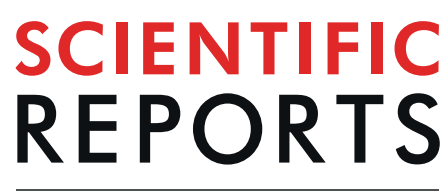

natureresearch

\title{
Prevalence and association of single nucleotide polymorphisms with sarcopenia in older women depends on definition
}

\author{
Praval Khanal $\mathbb{C}^{1,2^{*}}$, Lingxiao He $\mathbb{1}^{1,2}$, Georgina Stebbings ${ }^{1}$, Gladys L. Onambele-Pearson $\mathbb{1}^{1}$, \\ Hans Degens ${ }^{3,4,5}$, Alun Williams $\mathbb{1}^{1,6}$, Martine Thomis ${ }^{2}$ \& Christopher I. Morse ${ }^{1}$
}

The prevalence of sarcopenia depends on the definition used. There are, however, consistent sarcopenic characteristics, including a low muscle mass and muscle strength. Few studies have investigated the relationship between sarcopenia and genotype. A cross-sectional study was conducted with 307 community-dwelling $\geq 60$-year-old women in South Cheshire, UK. Handgrip strength was assessed with a handgrip dynamometer and skeletal muscle mass was estimated using bioelectrical impedance. DNA was extracted from saliva ( $38 \%)$ or blood ( $62 \%)$ and 24 single-nucleotide polymorphisms (SNPs) were genotyped. Three established sarcopenia definitions - \%Skeletal Muscle Mass (\%SMM), Skeletal Muscle Mass Index (SMI) and European Working Group on Sarcopenia in Older People (EWGSOP) - were used to assess sarcopenia prevalence. Binary logistic regression with age as covariate was used to identify SNPs associated with sarcopenia. The prevalence of sarcopenia was: \%SMM 14.7\%, SMI 60.6\% and EWGSOP 1.3\%. Four SNPs were associated with the \%SMM and SMI definitions of sarcopenia; FTO rs9939609, ESR1 rs4870044, NOS3 rs1799983 and TRHR rs7832552. The first three were associated with the \%SMM definition, and TRHR rs7832552 with the SMI definition, but none were common to both sarcopenia definitions. The gene variants associated with sarcopenia may help proper counselling and interventions to prevent individuals from developing sarcopenia.

Sarcopenia is defined as an ageing-related loss of both muscle mass and strength below a threshold level ${ }^{1}$. It is an important predictor of adverse outcomes such as limited mobility, increased risk of falls, decreased quality of life (QoL), hospitalization and mortality, and contributes to tens of millions of pounds of health care costs in the $\mathrm{UK}^{1-3}$. Although muscle weakness and skeletal muscle atrophy are overt characteristics of this geriatric syndrome, there is ongoing debate on the operational definition, screening and diagnosis, and optimal management and treatment of the condition ${ }^{1,4-6}$. The considerable heterogeneity in the reported prevalence of sarcopenia is largely attributable to the different definitions or cut-offs used ${ }^{7-9}$.

The fact that some elderly do not show sarcopenia, whilst others of the same age do ${ }^{10,11}$, suggests that some individuals are more susceptible to sarcopenia than others. The different susceptibility is likely due to a combination of factors including physical activity, diet, sedentary behaviour and genetics ${ }^{12-15}$. Several studies have reported an association of single nucleotide polymorphisms (SNPs) with lean mass, muscle volume and muscle strength ${ }^{16-18}$. It is thus possible that individuals carrying favourable gene variants are less susceptible to sarcopenia and hence can maintain independence until later life. To date, five studies have investigated the association of SNPs with sarcopenia; limited to VDR, IL6, ACTN3 and MSTN polymorphisms ${ }^{19-23}$. The studies identified an association of $A C T N 3$ and VDR gene variants with sarcopenia, but did not find any association with IL6 and MSTN variants. Unlike the previous studies that used low appendicular skeletal muscle mass as the cut-off for sarcopenia $^{19,20,22}$, the later studies used both low muscle mass and muscle function to define sarcopenia ${ }^{21,23}$.

\footnotetext{
${ }^{1}$ Department of Sport and Exercise Sciences, Musculoskeletal Science and Sports Medicine Research Centre, Manchester Metropolitan University, Manchester, UK. ²Department of Movement Sciences, Physical Activity, Sports \& Health Research Group, KU Leuven, Leuven, Belgium. ${ }^{3}$ Department of Life Sciences, Manchester Metropolitan University, Manchester, UK. ${ }^{4}$ Institute of Sport Science and Innovations, Lithuanian Sports University, Kaunas, Lithuania. ${ }^{5}$ University of Medicine and Pharmacy of Targu Mures, Targu Mures, Rumania. ${ }^{6}$ Institute of Sport, Exercise and Health, University College London, London, UK. *email: praval.khanal@gmail.com
} 


\begin{tabular}{|c|c|c|c|c|c|c|c|}
\hline \multirow{2}{*}{$\begin{array}{l}\text { Sarcopenia } \\
\text { definitions } \\
\text { (in columns) } \\
\text { |Variables (in } \\
\text { rows) }\end{array}$} & \multirow[b]{2}{*}{$\begin{array}{l}\text { General } \\
\text { characteristics } \\
(\mathbf{n}=\mathbf{3 0 7})\end{array}$} & \multicolumn{2}{|l|}{ SMI } & \multicolumn{2}{|l|}{$\%$ SMM } & \multicolumn{2}{|l|}{ EWGSOP } \\
\hline & & $\begin{array}{l}S \\
186(60.6 \%)\end{array}$ & NS & $\begin{array}{l}S \\
45(14.7 \%)\end{array}$ & NS & $\begin{array}{l}S \\
4(1.3 \%)\end{array}$ & NS \\
\hline Age (years) & $70.7 \pm 5.7$ & $71.0 \pm 5.3$ & $70.3 \pm 6.3$ & $71.6 \pm 5.6$ & $70.6 \pm 5.7$ & $76.8 \pm 7.0^{*}$ & $70.6 \pm 5.7$ \\
\hline Body Mass (kg) & $66.3 \pm 11.3$ & $63.4 \pm 9.3 * *$ & $70.9 \pm 12.5$ & $77.4 \pm 13.1 * *$ & $64.4 \pm 9.8$ & $68.5 \pm 10.9$ & $66.3 \pm 11.3$ \\
\hline BMI $\left(\mathrm{kg} / \mathrm{m}^{2}\right)$ & $25.9 \pm 4.2$ & $24.7 \pm 3.3^{* *}$ & $27.8 \pm 4.7$ & $30.2 \pm 5.5^{* *}$ & $25.2 \pm 3.4$ & $28.2 \pm 2.9$ & $25.9 \pm 4.2$ \\
\hline HGS (kg) & $29.9 \pm 5.0$ & $29.1 \pm 4.4^{* *}$ & $31.1 \pm 5.6$ & $28.4 \pm 4.9^{*}$ & $30.2 \pm 5.0$ & $17.8 \pm 2.2 * *$ & $30.0 \pm 4.9$ \\
\hline SMI $\left(\mathrm{kg} / \mathrm{m}^{2}\right)$ & $6.56 \pm 0.81$ & $6.05 \pm 0.51^{* *}$ & $7.33 \pm 0.53$ & $6.04 \pm 0.92 * *$ & $6.64 \pm 0.75$ & $6.29 \pm 0.18$ & $6.56 \pm 0.81$ \\
\hline
\end{tabular}

Table 1. Population and sarcopenia group characteristics with different sarcopenia definition. *And ** denote difference from non-sarcopenia group at $\mathrm{p}<0.05$ and $\mathrm{p}<0.001$ respectively. BMI, Body Mass Index, HGS, Hand Grip Strength, SMI, Skeletal Muscle Mass Index, S- Sarcopenia group, NS- Non sarcopenia group, n (\%), number (percentage) of sarcopenic individuals.

The genetic (as opposed to environmental) component of inter-individual differences in muscle size/strength are substantial, both in younger ${ }^{24,25}$ and older adults ${ }^{26}$. To date however, research in older adults has attempted to identify the precise genetic variations responsible for sarcopenia through four candidate SNPs, and none has compared different sarcopenia definitions in that regard. It would be advantageous to do so, with desirable outcomes including a greater understanding of the differences observed and the possibility of targeting interventions at those most at risk of sarcopenia. Considering the possible association of gene variants with sarcopenia (although the results may vary with different sarcopenia definitions) and heterogeneity in sarcopenia prevalence, the objectives of the present study were to assess 1) the prevalence of sarcopenia in a population of older women according to three different definitions of sarcopenia and 2) the association between sarcopenia and more SNPs than studied previously, for each definition of sarcopenia. The three chosen definitions were: skeletal muscle mass (SMM)/body mass (\%SMM) (sarcopenia defined to individuals with \%SMM $<22.1 \%)^{27}$, SMM/height ${ }^{2}$ (SMI) (sarcopenia defined to individuals with $\left.\mathrm{SMI} \leq 6.76 \mathrm{~kg} / \mathrm{m}^{2}\right)^{28}$ and SMI and Handgrip strength (HGS) cut-offs as suggested by European Working Group on Sarcopenia in Older People (EWGSOP) (sarcopenia defined to individuals with $\mathrm{SMI}<6.76 \mathrm{~kg} / \mathrm{m}^{2}$ and $\left.\mathrm{HGS}<20 \mathrm{~kg}\right)^{1}$.

\section{Results}

Prevalence of sarcopenia with different sarcopenia definitions. The prevalence of sarcopenia according to each definition was: \%SMM 14.7\%, SMI 60.6\% and EWGSOP 1.3\%. The general characteristics of the participants with each definition are presented in Table 1. Individuals identified as sarcopenic with EWGSOP were also sarcopenic by the SMI definition, while there was a discrepancy in individuals identified as sarcopenic between the SMI and \%SMM definitions: 34 elderly women were sarcopenic using both the \%SMM and SMI definitions, but 11 were sarcopenic using only the \%SMM definition and 152 were sarcopenic using only the SMI definition. Similarly, two individuals were common to both \%SMM and EWGSOP definitions, but 2 were with the EWGSOP only and 43 using only the \%SMM definition.

Associations of SNPs with sarcopenia according to the different definitions. All genotypes were in Hardy Weinberg equilibrium ( $\mathrm{p}>0.05$ ) (presented in Supplementary Table S1). The distribution of genotypes varies with the sarcopenia definitions (presented for FTO rs9939609 in Fig. 1).

Based on the \%SMM definition, the SNPs FTO rs9939609, ESR1 rs4870044 and NOS3 rs1799983 were associated with sarcopenia (presented in Table 2). Binary logistic regression, using age as a covariate, indicated that FTO rs9939609 AA homozygotes had 3.04 times higher risk of being sarcopenic than T-allele carriers $(\mathrm{OR}=3.037$, $95 \%$ confidence interval $[\mathrm{CI}]=1.439-6.411, \mathrm{p}=0.004)$. ESR $1 \mathrm{rs} 4870044 \mathrm{~T}$-allele carriers had a 2.54 times greater risk of being sarcopenic than CC homozygotes $(\mathrm{OR}=2.543,95 \% \mathrm{CI}=1.273-5.079, \mathrm{p}=0.008)$. Similarly, NOS3 rs 1799983 GG homozygotes had 2.26 times greater risk of being sarcopenic than T-allele carriers $(\mathrm{OR}=2.257$, $95 \% \mathrm{CI}=1.165-4.373, \mathrm{p}=0.016)$.

Based on the SMI definition, elderly females with the TRHR rs7832552 C-allele carriers had 2.6-fold higher risk of being sarcopenic when compared with TT homozygotes $(\mathrm{OR}=2.568,95 \% \mathrm{CI}=1.268-5.203, \mathrm{p}=0.009)$.

Using the EWGSOP definition, only four participants were defined as sarcopenic, therefore comparisons of genetic characteristics between sarcopenic and non-sarcopenic groups could not be performed.

None of the other 20 SNPs were associated with sarcopenia using any definition.

\section{Discussion}

The current study identified that different definitions of sarcopenia result in a widely different prevalence of sarcopenia, ranging from $1.3 \%$ to $60.6 \%$ in the present population of women aged $>60$ years. Three SNPs were associated with sarcopenia based on the \%SMM definition, and one SNP when using the SMI definition.

The heterogeneity of the prevalence of sarcopenia ( $1.3 \%$ to $60.6 \%)$, depending on sarcopenia definition used, is consistent with a previous study ${ }^{8}$. This confirms that the criteria, definition and threshold has a dramatic impact on the observed prevalence of sarcopenia, even in the same study population. Defining sarcopenia using \%SMM resulted in a prevalence of $14.7 \%$ in the present elderly female population. This prevalence falls within the range in previous studies using the same approach. For example, in the large population NHANES III study $10 \%$ of US women above 60 years ${ }^{27}$ and $23.6 \%$ of elderly French women ${ }^{29}$ were sarcopenic according to this definition. 

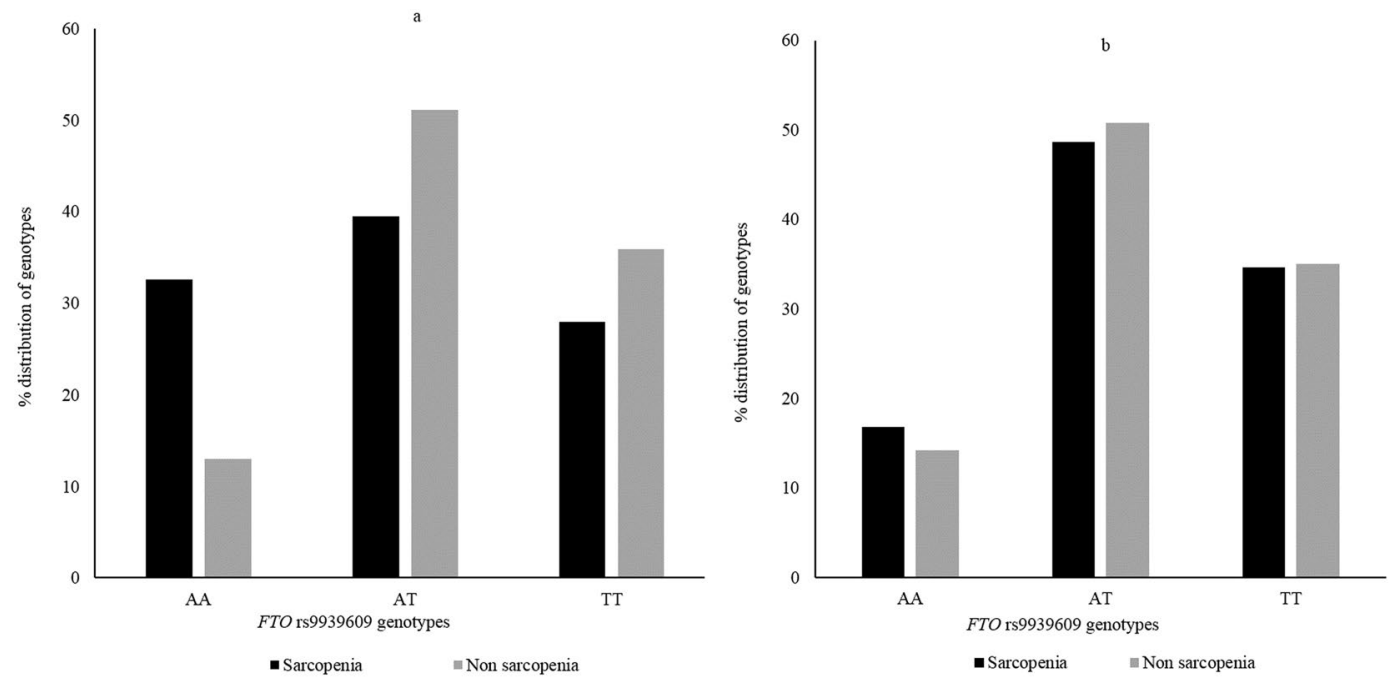

Figure 1. Distribution of FTO rs 9939609 genotypes between sarcopenia and non-sarcopenia groups with sarcopenia definitions (a) \%SMM and (b) SMI. Using \%SMM, AA homozygotes had over three times higher risk of being sarcopenic than T-allele carriers $(\mathrm{OR}=3.04, \mathrm{p}=0.004)$.

\begin{tabular}{|c|c|c|c|c|c|c|c|c|}
\hline $\begin{array}{l}\text { Sarcopenia } \\
\text { definitions }\end{array}$ & Variables & B & S.E $(\beta)$ & Wald's $\chi^{2}$ & $\mathbf{p}$ & OR & $95 \% \mathrm{CI}$ & Risk genotypes \\
\hline \multirow{3}{*}{ SMI } & Age & -0.260 & 0.021 & 1.463 & 0.226 & 0.975 & $0.935-1.016$ & \multirow{3}{*}{$\mathrm{CC}+\mathrm{CT}$} \\
\hline & TRHR rs7832552 & 0.943 & 0.360 & 6.858 & $0.009^{*}$ & 2.568 & $1.268-5.203$ & \\
\hline & PASE & $<0.001$ & 0.002 & 0.005 & 0.944 & 1.000 & $0.996-1.005$ & \\
\hline \multirow{9}{*}{$\% \mathrm{SMM}$} & Age & -0.032 & 0.029 & 1.268 & 0.260 & 0.968 & $0.915-1.024$ & \multirow{3}{*}{ AA } \\
\hline & FTO rs9939609 & 1.111 & 0.381 & 8.492 & $0.004^{*}$ & 3.037 & $1.439-6.411$ & \\
\hline & PASE & 0.008 & 0.004 & 3.735 & 0.053 & 1.008 & $1.000-1.015$ & \\
\hline & Age & -0.031 & 0.028 & 1.222 & 0.269 & 0.970 & $0.919-1.024$ & \multirow{3}{*}{ GG } \\
\hline & NOS3 rs1799983 & 0.814 & 0.337 & 5.820 & $0.016^{*}$ & 2.257 & $1.165-4.373$ & \\
\hline & PASE & 0.009 & 0.004 & 4.814 & 0.028 & 1.009 & $1.001-1.016$ & \\
\hline & Age & -0.031 & 0.028 & 1.308 & 0.253 & 0.969 & $0.918-1.023$ & \multirow{3}{*}{$\mathrm{TT}+\mathrm{CT}$} \\
\hline & ESR1 rs4870044 & 0.933 & 0.353 & 6.988 & $0.008^{*}$ & 2.543 & $1.273-5.079$ & \\
\hline & PASE & 0.009 & 0.004 & 4.787 & $0.029 *$ & 1.009 & $1.001-1.016$ & \\
\hline
\end{tabular}

Table 2. Binary logistic regression model of single nucleotide polymorphisms with age and PASE on sarcopenia. *Denotes association of SNPs and sarcopenia in a model.

The SMI definition of sarcopenia has previously resulted in prevalence ranging from $2.8 \%-42.0 \%{ }^{29-31}$. At the extremes, this lower prevalence in sarcopenia is due to threshold levels being set arguably too conservatively (SMI $6.2 \mathrm{~kg} / \mathrm{m}^{2}$ previously $)^{29}$, compared to $6.76 \mathrm{~kg} / \mathrm{m}^{2}$ (SMI) in the present study definition consistent with previous work $^{28}$. The prevalence of sarcopenia (1.3\%) in elderly females in the present study using the EWGSOP definition is consistent with studies on different populations - for instance, $2.5 \%$ in Taiwanese ${ }^{32}, 4.5 \%$ in German $^{33}$ and $6.8 \%$ in Japanese ${ }^{34}$ women. It should be noted that higher prevalences have been reported, ranging between $22-48 \%$ in some of the studies conducted across Europe, Asia and South America ${ }^{35-39}$, but these higher prevalances tend to be in populations incorporating the oldest old ( $>70$ years) or those not living independently and/or their own young reference group (rather than cut offs).

Within this study, elderly women identified as sarcopenic using the EWGSOP definition were also sarcopenic using the SMI definition. The SMI definition used $\leq 6.76 \mathrm{~kg} / \mathrm{m}^{2}$ as the cut-off for low muscle mass in defining sarcopenia, while EWGSOP identified the sarcopenic with the same cut-off for low muscle mass and additionally $<20 \mathrm{~kg}$ handgrip strength that evidently made no material difference in our cohort. However, the discrepancy in those identified as sarcopenic between those two definitions and the \%SMM definition could be because the \%SMM definition uses only values of mass while the other two both adjust values of mass according to height. The difference in the prevalence of sarcopenia by considering height and body mass as normalizing factors has been observed in a previous study ${ }^{40}$. These observations clearly indicate that a consensus is needed concerning the definition of sarcopenia.

The present study identified FTO rs9939609 AA homozygotes to be at over 3-fold higher risk for sarcopenia compared to T-allele carriers. This genotype has been previously associated as a risk genotype for obesity-related indices such as increased BMI and fat mass $^{41-43}$, and clinical conditions such as type 2 diabetes ${ }^{44}$ 
and tuberculosis ${ }^{45}$. The importance of FTO during skeletal muscle development and differentiation was reflected by the impaired skeletal muscle development in FTO-deficient mice, due to myogenic suppression ${ }^{46}$. There was an association between FTO rs9939609 AA and lean body mass; however, that association disappeared when controlling for fat mass ${ }^{41,47}$. Recent studies have reported over-representation of FTO rs9939609 AA genotypes in heavier athletes ${ }^{48,49}$, however, none has investigated how the body mass and muscle mass are affected by this genotype in those athletic populations. We previously demonstrated that the T-allele is associated with higher appendicular and arm lean mass in untrained young $\mathrm{men}^{49}$. It therefore appears that FTO AA-genotype individuals have lower skeletal muscle mass and are at higher risk of sarcopenia.

The current study is the first to identify the NOS3 rs1799983 GG genotype as a risk factor for sarcopenia, with over 2-fold higher risk compared to T-allele carriers, for sarcopenia in the elderly. This finding is consistent with previous studies that have reported the T allele as more frequent in power oriented athletes ${ }^{50}$ and female short distance swimmers ${ }^{51}$ and association of TT homozygote with absolute gain in dynamic strength following resistance training in women ${ }^{52}$. Biologically, the NOS3 gene encodes the enzyme endothelial nitric oxide synthase (eNOS) that catalyses the synthesis of nitric oxide (NO). NO has been identified as a determinant of individual variations in health and exercise related phenotypes ${ }^{53}$, mitochondrial energy production ${ }^{54}$ and muscle hypertrophy ${ }^{55}$. The NOS3 rs1799983 T allele has been associated with higher NOS activity compared to the G-allele ${ }^{56}$. The association of the NOS3 rs1799983 T allele with a higher NOS activity and the beneficial role of NO in skeletal muscle, particularly muscle hypertrophy and energy production, provide a potential basis for the present observation that elderly women who are GG homozygotes are at higher risk of being sarcopenic.

The current study also identified the ESR 1 rs4870044 T allele as a risk factor, with 2.5-fold higher risk of sarcopenia in T-allele carriers compared to CC homozygotes. Biologically, ESR1 encodes the oestrogen receptor protein that interacts with oestrogen and plays an important role in growth of bone and maintenance of bone mass. ESR1 is also expressed in human skeletal muscle ${ }^{57}$. However, the role of oestrogen in skeletal muscle is still to be elucidated. The ESR1 rs4870044 T allele has been associated previously with low bone mineral density (BMD) ${ }^{58,59}$ and a higher risk of hip fracture ${ }^{60}$. Since ageing results in the deterioration of both bone and muscle, and bone geometry is partly determined by muscle mass/strength ${ }^{61,62}$, ESR1 might contribute to both muscle and bone phenotypes $^{63}$. Although previous studies did not find an association between rs4870044 and muscle strength ${ }^{64-66}$, we suggest that the discrepancy between those studies and our study might be attributable to us defining sarcopenia by the \%SMM while the other studies assessed the relationship with muscle strength. Whatever the cause of the discrepancy, the higher risk of sarcopenia in T-allele carriers may be related to the physiological activities of oestrogen, mediated by oestrogen receptors ${ }^{67,68}$ where lower levels of oestrogen have previously been associated with low bone mass and bone mineral density ${ }^{69,70}$, as well as low muscle mass ${ }^{71}$ and muscle strength ${ }^{71,72}$.

In the current study, TRHR rs7832552 C-allele carriers were at over 2-fold higher risk compared to TT homozygotes for sarcopenia defined according to the SMI. This aligns with studies associating the T-allele with increased muscle mass. For example, a genome-wide association study identified individuals homozygous for the TRHR rs7832552 T-allele to have on average $2.5 \mathrm{~kg}$ more lean body mass than heterozygotes and C-allele homozygotes $^{73}$. Similarly, this polymorphism has been associated with sprint/power performance ${ }^{74}$. A greater sarcopenic risk for C-allele carriers in the present elderly might be explained by lower TRHR expression ${ }^{75}$ and hence impaired action of thyroid hormone that is considered important to preserve muscle strength ${ }^{76}$.

The prevalence and associations of sarcopenia and SNPs were studied with three different sarcopenia definitions. One limitation is that the current study was limited to older women only, but then particularly women suffer from (the consequence of) sarcopenia. Another limitation is that SMM was estimated using BIA instead of the more accurate MRI or DEXA. Although many studies have shown a good agreement (e.g. standard error of estimate $2.7 \mathrm{~kg}^{77}$ ) between BIA and MRI/DEXA ${ }^{77-79}$, BIA might overestimate muscle mass ${ }^{80,81}$. Therefore, future studies that adopt a more rigorous approach to assessing muscle mass (MRI or DXA) may observe stronger SNP associations than those reported in the present study. As diet $^{82}$ also plays an important role in the maintenance of muscle mass/strength, we also encourage future work that incorporates diet and those additional possible confounders to understand the sarcopenia mechanism in depth.

Within our study, only four of the twenty-four investigated SNPs were associated with sarcopenia. Skeletal muscle mass and strength are highly polygenic in nature ${ }^{83}$, hence the influence of any single SNP on the risk of sarcopenia is probably limited. A polygenic approach combining multiple risk alleles for sarcopenia will ultimately capture a substantial portion of the genetic risk and any future practical tool will use that approach. However, many more individual SNPs first need to be identified before a polygenic approach becomes a worthwhile development. It should also be noted that the sarcopenia definitions identified different SNPs to be associated with sarcopenia. This is probably because different individuals were identified as sarcopenic by the different definitions and demonstrates how inconsistency in the definition of sarcopenia in the literature will have consequences for the ability to identify causative factors. Given the perpetual decline of muscle mass and strength in the elderly, although individuals in our study identified as sarcopenic using the SMI and \%SMM definitions (in fact they were independent and could perform daily activities without any limitations), they are probably at a higher risk of developing sarcopenia and doing so earlier than individuals not identified as sarcopenic using any definition. As a genetic association was found, SNP- related risk seems pertinent even in those elderly who are currently healthy. Similarly, the tested SNPs were not selected randomly, but due to their previous association with skeletal muscle phenotypes or similar phenotypes, so we believe the results observed are informative regarding sarcopenia-related phenotypes.

The prevalence of sarcopenia is to a large extent affected by the definition used, and ranged in our population of older women from 1.3 to $60.6 \%$. This clearly shows that, when comparing studies, due attention must be given to the definition of sarcopenia. We also identified four SNPs (FTO rs9939609, ESR1 rs4870044, NOS3 rs1799983 and TRHR rs7832552) associated with sarcopenia and this information might be used (with other data) to identify individuals at a higher risk of sarcopenia and facilitate early targeted intervention to offset that higher risk. 


\begin{abstract}
Methods
Participants. Participants comprised 60- to 91-year-old Caucasian women $(\mathrm{n}=307 ; 70.7 \pm 5.7$ years, $66.3 \pm 11.2 \mathrm{~kg}, 1.60 \pm 0.06 \mathrm{~m}$; mean $\pm \mathrm{SD}$ ). All participants were recruited from social groups, and subsequent word-of-mouth between participants. These social groups ensured the participants were either physically active at a recreational level (e.g. table tennis, walking groups, dance classes) and societally engaged (e.g. book groups, and discussion groups). All were independently living, ambulatory and had no history of severe muscle and bone issues such as osteoporosis or rheumatoid arthritis, nor cancer, cardiovascular-related diseases or nervous system disorders such as Alzheimer's, convulsions or epilepsy.
\end{abstract}

Skeletal muscle mass index. Skeletal muscle mass was quantified using Bioelectrical Impedance Analysis (BIA) (Model 1500; Bodystat, Isle of Man, UK). Although Dual-Energy X-ray Absorptiometry (DEXA) is commonly used for measuring appendicular lean mass index (ALMI) in sarcopenia studies ${ }^{8,84}$, BIA has been suggested as a valid and low-cost alternative for measuring Skeletal Muscle Mass Index (SMI) and is a common tool in larger population studies ${ }^{27,85}$. Indeed, a high correlation between muscle mass measured with BIA and DEXA has been reported ${ }^{78,79}$. Participants were instructed to lie on a physiotherapist bed in a supine position with both upper and lower limbs slightly abducted from the body for about 4-5 min. Two adhesive electrodes were placed on the dorsum of the hand and foot on the right side of the body. An electrical current (frequency: $50 \mathrm{kHz}$; amplitude: $0.4 \mathrm{~mA}$ ) was then passed between these electrodes and the skeletal muscle mass was estimated using an established formula ${ }^{77}$ :

$$
\text { Skeletal Muscle Mass }(\mathrm{SMM})=\left[\mathrm{Ht}^{2} /(\mathrm{R} \times 0.401)+(\operatorname{sex} \times 3.825)+(\operatorname{age} \times-0.071)\right]+5.102
$$

Where $\mathrm{Ht}$ is height in $\mathrm{cm}, \mathrm{R}$ is resistance in $\Omega$ and age in years. For sex, a male is scored as 1 and female as 0 . SMI as $\mathrm{SMM} / \mathrm{Ht}^{2}$ was calculated using $\mathrm{SMM}$ in $\mathrm{kg}$ and height of the participant in $\mathrm{m}$.

Handgrip strength. Handgrip strength (HGS) was measured using a digital load cell handgrip dynamometer (JAMAR plus, JLW Instruments, Chicago, USA) with a previously validated protocol ${ }^{86}$. Participants were instructed to stand in an upright position with the dynamometer held with the arm straight, and flexed at $90^{\circ}$ to the shoulder. Verbal encouragement was provided to each participant to squeeze the handgrip dynamometer with maximum force, which was maintained for $5 \mathrm{~s}$. The left and right arm were alternated, with 1 min between trials. The highest grip strength of three maximal efforts on each arm was recorded. The test-retest reliability of HGS in the dominant hand is high $(\mathrm{ICC}=0.99)$ in healthy elderly participants ${ }^{87}$.

Physical activity scale for elderly questionnaire. Participants completed the Physical Activity Scale for Elderly (PASE) questionnaire in the lab on the testing day. The questionnaire is a 7-day recall questionnaire identifying time spent undertaking activities such as sitting, moderate intensity activities, recreational activities, strenuous activities and endurance and muscle strength related exercises. The questionnaire includes questions related to time spent in household work, gardening, caring for a dependent person, and work (paid or voluntary). The total PASE score was computed by multiplying the amount of time spent in each activity (hours/week) or participation (yes/no) in an activity by the empirically derived item weights and summing over all activities ${ }^{88}$.

DNA sample collection, DNA extraction, SNPs selection and genotyping. Two techniques for DNA collection were adopted. All participants were encouraged to provide a forearm venous blood sample, of whom 189 did so. If participants were unwilling to provide a blood sample or there was difficulty in obtaining a blood sample, a saliva sample was collected (116 participants). It was not possible to collect either sample from two participants. Genotyping was successful for $99.9 \%$ of the sample-SNPs combinations $(7,313$ out of 7,320$)$; four different assays did not work for four samples, and three for one sample.

Blood $(5 \mathrm{~mL})$ was collected from a superficial forearm vein in 5-mL EDTA tubes (BD Vacutainer Systems, Plymouth, UK). Samples were stored at $-20^{\circ} \mathrm{C}$ until further processing. Superfluous saliva samples were collected using Oragene DNA OG-500 collection tubes (DNA Genotek Inc., Ontario, Canada) according to the manufacturer's instructions. Saliva samples were stored at room temperature until DNA extraction. Genomic DNA was extracted from both blood and saliva samples using a QIAcube, QIAamp DNA Blood Mini kit and standard spin column protocol (Qiagen, Crawley, UK).

Extracted DNA samples were genotyped for 24 polymorphisms selected based on the literature on the previous associations of those SNPs with relevant phenotypes, previous or hypothesised associations with sarcopenia and understood functional relevance. An initially 36 candidate SNPs identified were then reduced to 24 considering the number of studies reporting each association, the presence of conflicting results and the known transcriptional difference for some of the SNPs. Associations of the SNPs with previously reported relevant phenotypes are presented in Supplementary Table S2.

Two techniques were adopted for genotyping. The Fluidigm EP1 system (Fluidigm, Cambridge, UK) was used initially, but where errors occurred $(\sim 1 \%)$, such as when duplicate samples were not in agreement, a second run was performed using StepOnePlus real-time PCR (Applied Biosystems ${ }^{\circledR}$, Paisley, UK).In brief, four runs were performed, and genotype was determined using Fluidigm 192.24 Dynamic Array IFC (Integrated Fluidic Circuit, Fluidigm) in accordance with the manufacturer's instructions. Each assay $(4 \mu \mathrm{L})$ comprised $2.0 \mu \mathrm{L}$ of assay loading reagent [2X] (Fluidigm), 1.0 $\mu \mathrm{L}$ SNP genotyping Assay Mix [40X] (Applied Biosystems ${ }^{\circledR}$ ), $0.2 \mu \mathrm{L}$ ROX [50X] (Invitrogen, Carlsbad, CA) and $0.8 \mu \mathrm{L}$ DNA-free water (Qiagen). Each sample (4 $\mu \mathrm{L})$ contained $1.6 \mu \mathrm{L}$ genomic DNA, 2.0 $\mu \mathrm{L}$ GTXpress master mix [2X] (Applied Biosystems ${ }^{\circledR}$, PN 4401892), 0.2 $\mu \mathrm{L}$ Fast GT Sample Loading Reagent [20X] (Fluidigm, PN 100-3065), and 0.2 $\mu$ L DNA-free water. No-template controls (NTCs) were included in each run. Each of the assays $(3.75 \mu \mathrm{L})$ and samples $(4 \mu \mathrm{L})$ were pipetted into separate inlets of the chip according to manufacturer's instructions. Assays and samples were loaded and mixed using the Integrated IFC 
Controller RX software. The chip was then loaded into a thermal cycler (FC1 Fluidigm, PN 100-1279 D1) and the GT 192.24 Fast v1.pcl protocol was run. The thermocycling protocol was: an initial $120 \mathrm{~s}$ at $95^{\circ} \mathrm{C}$ followed by 45 cycles of denaturation for $2 \mathrm{~s}$ at $95^{\circ} \mathrm{C}$ and then annealing and extension for $20 \mathrm{~s}$ at $60^{\circ} \mathrm{C}$. TaqMan assays included $\mathrm{VIC}^{\circledR}$ and FAM ${ }^{\circledR}$ dyes for all SNPs and genotypes were identified based on end-point fluorescence (https://www. thermofisher.com/np/en/home.html) (attached in Supplementary Table S3). When using the StepOnePlus, the reaction volume was $10 \mu \mathrm{L}$ that contained $0.2 \mu \mathrm{L}$ DNA, $5 \mu \mathrm{L}$ GTXpress master mix, $4.3 \mu \mathrm{L}$ nuclease-free $\mathrm{H}_{2} \mathrm{O}$ and $0.5 \mu \mathrm{L}$ TaqMan SNP genotyping assay [20X]. An initial $20 \mathrm{~s}$ at $95^{\circ} \mathrm{C}$ was followed by 50 cycles of denaturation for $3 \mathrm{~s}$ at $95^{\circ} \mathrm{C}$, then annealing and extension for $20 \mathrm{~s}$ at $60^{\circ} \mathrm{C}$. Genotypes were identified based on reporter dyes $\mathrm{VIC}^{\circledR}$ and $\mathrm{FAM}^{\circledR}$ intensity and visualized using cluster plots. Except for PTK2 rs7460, for which nucleotides were reported on the reverse strand according to NCBI, others were reported on the forward strand (shown in Supplementary Table S3). All samples were analysed in duplicate and 100\% agreement was required to minimise genotyping error ${ }^{89}$.

Assessment of sarcopenia. Sarcopenia was assessed using three different definitions. The first definition, \%SMM, was calculated as SMM/body mass*100. Participants were defined as sarcopenic if $\% \mathrm{SMM}<22.1 \%{ }^{27}$. The second definition, SMI, previously used by Janssen et al. ${ }^{28}$, was calculated as SMM $/$ height ${ }^{2}$. Participants were defined as sarcopenic if SMI $\leq 6.76 \mathrm{~kg} / \mathrm{m}^{228}$. Definition three used the measures of low SMI and low HGS as suggested by the EWGSOP; for which individuals with both $\mathrm{SMI}<6.76 \mathrm{~kg} / \mathrm{m}^{2}$ and a $\mathrm{HGS}<20 \mathrm{~kg}$ were considered sarcopenic ${ }^{1}$.

Statistical analysis. The Kolmogorov-Smirnov test was used to assess whether the data had a normal distribution and Levene's test was used to assess the homogeneity of variance of HGS and SMI. The frequency distribution of each SNP was assessed for Hardy-Weinberg equilibrium (HWE) using chi-square tests. Binary logistic regression was performed to investigate the association of sarcopenia and the SNPs studied, with age and PASE score used as covariate. The analysis was performed separately for each individual SNP. In instances where the number of homozygous participants was low, the homozygous group was combined with the heterozygous group and a two-group analysis was performed. $\mathrm{p}<0.05$ was considered statistically significant. Where there was an association or tendency of an association $(0.05<\mathrm{p}<0.15)^{90,91}$, the homozygous groups were combined with the heterozygous group in a recessive and dominant model and then the analyses were re-run. Odds ratios (OR) for the risk genotype for sarcopenia were estimated for each SNP. Benjamini-Hochberg correction was performed to reduce the chance of type I error for multiple testing ${ }^{92}$ with 24 tests and False Discovery Rate of 0.25 . All the tests were performed in SPSS Version 26.0.

Ethics statement. Study protocols were in accordance with the guidelines of the Declaration of Helsinki (World Medical Association, 2013) and approved by the Ethics Committee of Manchester Metropolitan University. Informed written consent was obtained from all participants prior to involvement in the study.

\section{Data availability}

The datasets analysed during the present study are available on reasonable request from corresponding author.

Received: 30 September 2019; Accepted: 27 January 2020;

Published online: 19 February 2020

\section{References}

1. Cruz-Jentoft, A. J. et al. Sarcopenia: European consensus on definition and diagnosisReport of the European Working Group on Sarcopenia in Older People. Age ageing 39, 412-423 (2010).

2. McNamee, P., Bond, J. \& Buck, D. Resource Implications Study of the Medical Research Council Cognitive, F. \& Ageing, S. Costs of dementia in England and Wales in the 21st century. Br. J. Psychiatry 179, 261-266 (2001).

3. Fahy, N. Who is shaping the future of European health systems? BMJ 344, e1712 (2012).

4. Cederholm, T., Cruz-Jentoft, A. J. \& Maggi, S. Sarcopenia and fragility fractures. Eur. J. Phys. Rehabil. Med. 49, 111-117 (2013).

5. Dent, E. et al. International Clinical Practice Guidelines for Sarcopenia (ICFSR): Screening, Diagnosis and Management. J. Nutr. Health Aging 22, 1148-1161, https://doi.org/10.1007/s12603-018-1139-9 (2018).

6. Fielding, R. A. et al. Sarcopenia: an undiagnosed condition in older adults. Current consensus definition: prevalence, etiology, and consequences. International working group on sarcopenia. J. Am. Med. Dir. Assoc. 12, 249-256, https://doi.org/10.1016/j. jamda.2011.01.003 (2011).

7. Beaudart, C. et al. Estimation of sarcopenia prevalence using various assessment tools. Exp. gerontology 61, 31-37, https://doi. org/10.1016/j.exger.2014.11.014 (2015).

8. Bijlsma, A. Y. et al. Defining sarcopenia: the impact of different diagnostic criteria on the prevalence of sarcopenia in a large middle aged cohort. Age. 35, 871-881, https://doi.org/10.1007/s11357-012-9384-z (2013).

9. Pagotto, V. \& Silveira, E. A. Methods, diagnostic criteria, cutoff points, and prevalence of sarcopenia among older people. Sci. World J. 2014, 1-11, https://doi.org/10.1155/2014/231312 (2014).

10. Isanejad, M. et al. Dietary protein intake is associated with better physical function and muscle strength among elderly women. $B r$. J. Nutr. 115, 1281-1291, https://doi.org/10.1017/S000711451600012X (2016).

11. Steffl, M. et al. Relationship between sarcopenia and physical activity in older people: a systematic review and meta-analysis. Clin. Interv. Aging 12, 835, https://doi.org/10.2147/CIA.S132940 (2017).

12. Carmelli, D. \& Reed, T. Stability and change in genetic and environmental influences on hand-grip strength in older male twins. J. Appl. Physiol. 89, 1879-1883, https://doi.org/10.1152/jappl.2000.89.5.1879 (2000).

13. de Camargo Smolarek, A. et al. Strength Decline in Sedentary Males and Females of Different Ages. J. Exerc. Physiol. Online 21, 40-50 (2018).

14. Bruce, S. Healthy diet and better muscle function and quality in older women. Age Ageing 46, 538-539, https://doi.org/10.1093/ ageing/afx036 (2017).

15. Gerdhem, P., Ringsberg, K. A. M., Obrant, K. J. \& Akesson, K. Association between 25-hydroxy vitamin D levels, physical activity, muscle strength and fractures in the prospective population-based OPRA Study of Elderly Women. Osteoporos. Int. 16, 1425-1431, https://doi.org/10.1007/s00198-005-1860-1 (2005). 
16. Charbonneau, D. E. et al. ACE genotype and the muscle hypertrophic and strength responses to strength training. Med. Sci. Sports Exerc. 40, 677-683, https://doi.org/10.1249/MSS.0b013e318161eab9 (2008).

17. Garatachea, N. \& Lucia, A. Genes, physical fitness and ageing. Ageing Res. Rev. 12, 90-102, https://doi.org/10.1016/j.arr.2012.09.003 (2013).

18. Tan, L. J., Liu, S. L., Lei, S. F., Papasian, C. J. \& Deng, H. W. Molecular genetic studies of gene identification for sarcopenia. Hum. Genet. 131, 1-31, https://doi.org/10.1007/s00439-011-1040-7 (2012).

19. Cho, J., Lee, I. \& Kang, H. ACTN3 Gene and Susceptibility to Sarcopenia and Osteoporotic Status in Older Korean Adults. Biomed. Res. Int. 2017, 4239648, https://doi.org/10.1155/2017/4239648 (2017).

20. Roth, S. M., Zmuda, J. M., Cauley, J. A., Shea, P. R. \& Ferrell, R. E. Vitamin D receptor genotype is associated with fat-free mass and sarcopenia in elderly men. J. Gerontology Ser. A: Biol. Sci. Med. Sci. 59, B10-B15 (2004).

21. Tasar, P. T. Retrospective investigation of Interleukin IL-1 and IL-6 genes polymorphism among elderly patients with sarcopenia in the Turkish population. Genet. Mol. Res. 17, gmr16039881 (2018).

22. Walsh, S., Ludlow, A. T., Metter, E. J., Ferrucci, L. \& Roth, S. M. Replication study of the vitamin D receptor (VDR) genotype association with skeletal muscle traits and sarcopenia. Aging Clin. Exp. Res. 28, 435-442, https://doi.org/10.1007/s40520-015-04478 (2016).

23. Tosun Tasar, P. et al. Myostatin Gene Polymorphism in an Elderly Sarcopenic Turkish Population. Genet. Test. Mol. Biomarkers 19, 457-460, https://doi.org/10.1089/gtmb.2015.0033 (2015).

24. Erskine, R. M., Jones, D. A., Maganaris, C. N. \& Degens, H. In vivo specific tension of the human quadriceps femoris muscle. Eur. J. Appl. Physiol. 106, 827, https://doi.org/10.1007/s00421-009-1085-7 (2009).

25. Stebbings, G. K., Morse, C. I., Williams, A. G., Day, S. H. Variability and distribution of muscle strength and its determinants in humans. Muscle Nerve 49, 879-886, https://doi.org/10.1002/mus.24075 (2014).

26. Chen, L., Nelson, D. R., Zhao, Y., Cui, Z. \& Johnston, J. A. J. B. g. Relationship between muscle mass and muscle strength, and the impact of comorbidities: a population-based, cross-sectional study of older adults in the United States. BMC Geriatr 13, 74, https:// doi.org/10.1186/1471-2318-13-74 (2013).

27. Janssen, I., Heymsfield, S. B. \& Ross, R. Low relative skeletal muscle mass (sarcopenia) in older persons is associated with functional impairment and physical disability. J. Am. Geriatrics Soc. 50, 889-896, https://doi.org/10.1046/j.1532-5415.2002.50216.x (2002).

28. Janssen, I., Baumgartner, R. N., Ross, R., Rosenberg, I. H. \& Roubenoff, R. Skeletal muscle cutpoints associated with elevated physical disability risk in older men and women. Am. J. Epidemiol. 159, 413-421, https://doi.org/10.1093/aje/kwh058 (2004).

29. Tichet, J. et al. Prevalence of sarcopenia in the French senior population. J. Nutr. Health Aging 12, 202-206 (2008).

30. Chien, M. Y., Huang, T. Y. \& Wu, Y. T. Prevalence of sarcopenia estimated using a bioelectrical impedance analysis prediction equation in community-dwelling elderly people in Taiwan. J. Am. Geriatrics Soc. 56, 1710-1715 (2008).

31. Janssen, I. Influence of sarcopenia on the development of physical disability: the Cardiovascular Health Study. J. Am. Geriatr. Soc. 54, 56-62, https://doi.org/10.1111/j.1532-5415.2005.00540.x (2006).

32. Wu, C. H. et al. Prevalence and associated factors of sarcopenia and severe sarcopenia in older $\mathrm{T}$ aiwanese living in rural community: The T ianliao O ld P eople study 04. Geriatrics gerontology Int. 14, 69-75 (2014).

33. Kemmler, W. et al. Prevalence of sarcopenia in Germany and the corresponding effect of osteoarthritis in females 70 years and older living in the community: results of the FORMoSA study. Clin. Interv. Aging 10, 1565-1573, https://doi.org/10.2147/CIA.S89585 (2015).

34. Yoshida, D. et al. Using two different algorithms to determine the prevalence of sarcopenia. Geriatrics gerontology Int. 14, 46-51 (2014).

35. Volpato, S. et al. Prevalence and clinical correlates of sarcopenia in community-dwelling older people: application of the EWGSOP definition and diagnostic algorithm. J. Gerontol. A Biol. Sci. Med. Sci 69, 438-446, https://doi.org/10.1093/gerona/glt149 (2014).

36. Yamada, M. et al. Prevalence of sarcopenia in community-dwelling Japanese older adults. J. Am. Med. Dir. Assoc. 14, 911-915, https://doi.org/10.1016/j.jamda.2013.08.015 (2013).

37. Arango-Lopera, V. E., Arroyo, P., Gutiérrez-Robledo, L. M. \& Pérez-Zepeda, M. U. Prevalence of sarcopenia in Mexico City. Eur. Geriatric Med. 3, 157-160 (2012).

38. ter Borg, S. et al. Differences in nutrient intake and biochemical nutrient status between sarcopenic and nonsarcopenic older adults-results from the Maastricht Sarcopenia Study. J. Am. Med. Dir. Assoc. 17, 393-401 (2016).

39. Velázquez Alva, M. D. C., Irigoyen Camacho, M. E., Delgadillo Velázquez, J. \& Lazarevich, I. The relationship between sarcopenia, undernutrition, physical mobility and basic activities of daily living in a group of elderly women of Mexico City. Nutricion hospitalaria 28, 514-521 (2013).

40. Kim, K. M., Jang, H. C. \& Lim, S. J. T. Kjoim Differences among skeletal muscle mass indices derived from height-, weight-, and body mass index-adjusted models in assessing sarcopenia. Korean J. Intern. Med. 31, 643 (2016).

41. Sonestedt, E. et al. Association between fat intake, physical activity and mortality depending on genetic variation in FTO. Int. J. Obes. 35, $1041(2011)$

42. Livshits, G., Malkin, I., Moayyeri, A., Spector, T. D. \& Hammond, C. J. Association of FTO gene variants with body composition in UK twins. Ann. Hum. Genet. 76, 333-341, https://doi.org/10.1111/j.1469-1809.2012.00720.x (2012).

43. Frayling, T. M. et al. A common variant in the FTO gene is associated with body mass index and predisposes to childhood and adult obesity. Sci. 316, 889-894 (2007).

44. Sabarneh, A. et al. Common FTO rs9939609 variant and risk of type 2 diabetes in Palestine. BMC Med. Genet. 19, 156, https://doi. org/10.1186/s12881-018-0668-8 (2018).

45. Feng, Y. et al. Obesity-associated gene FTO rs 9939609 polymorphism in relation to the risk of tuberculosis. BMC Infect. Dis. 14, 592, https://doi.org/10.1186/s12879-014-0592-2 (2014).

46. Wang, X. et al. FTO is required for myogenesis by positively regulating mTOR-PGC-1 $\alpha$ pathway-mediated mitochondria biogenesis. Cell death Dis. 8, e2702 (2017).

47. Jess, T. et al. Impact on weight dynamics and general growth of the common FTO rs9939609: a longitudinal Danish cohort study. Int. J. Obes. 32, 1388-1394, https://doi.org/10.1038/ijo.2008.110 (2008).

48. Guilherme, J. P. L. et al. The A-allele of the FTO Gene rs9939609 Polymorphism Is Associated With Decreased Proportion of Slow Oxidative Muscle Fibers and Over-represented in Heavier Athletes. J. Strength. Conditioning Res. 33, 691-700 (2019).

49. Heffernan, S. M. et al. Fat mass and obesity associated (FTO) gene influences skeletal muscle phenotypes in non-resistance trained males and elite rugby playing position. BMC Genet. 18, 4, https://doi.org/10.1186/s12863-017-0470-1 (2017).

50. Eider, J. et al. Endothelial nitric oxide synthase g894t (rs1799983) gene polymorphism in polish athletes. Open. Life Sci. 9, 260-267 (2014).

51. Zmijewski, P., Cieszczyk, P. \& Ahmetov, I. I. The NOS3 G894T (rs1799983) and-786T/C (rs2070744) polymorphisms are associated with elite swimmer status. Biol. Sport. 35, 313-319 (2018).

52. Guidry, M. A. et al. Endothelial Nitric Oxide Synthase (NOS3). ISRN Vascular Medicine 2012 (2012).

53. Bray, M. S. et al. The human gene map for performance and health-related fitness phenotypes: the 2006-2007 update. Med. Sci. Sports Exerc. 41, 35-73 (2009).

54. Brown, G. C. Mechanisms of inflammatory neurodegeneration: iNOS and NADPH oxidase. Biochem. Soc. Trans. 35, 1119-1121, https://doi.org/10.1042/BST0351119 (2007). 
55. Smith, L. W., Smith, J. D. \& Criswell, D. S. Involvement of nitric oxide synthase in skeletal muscle adaptation to chronic overload. J. Appl. Physiol. 92, 2005-2011, https://doi.org/10.1152/japplphysiol.00950.2001 (2002).

56. Persu, A. et al. Modifier effect of ENOS in autosomal dominant polycystic kidney disease. Hum. Mol. Genet. 11, 229-241, https://doi. org/10.1093/hmg/11.3.229 (2002).

57. Lemoine, S. et al. Estrogen receptor alpha mRNA in human skeletal muscles. Med. Sci. Sports Exerc. 35, 439-443, https://doi. org/10.1249/01.MSS.0000053654.14410.78 (2003).

58. Luo, L. et al. Association of ESR1 and C6orf97 gene polymorphism with osteoporosis in postmenopausal women. Mol. Biol. Rep. 41, 3235-3243, https://doi.org/10.1007/s11033-014-3186-6 (2014).

59. Martinaityte, I. et al. Bone mineral density is associated with vitamin D related rs6013897 and estrogen receptor polymorphism rs4870044: The Tromsø study. PLoS one 12, e0173045 (2017).

60. Hidalgo-Bravo, A. et al. Association of RMND1/CCDC170-ESR1 single nucleotide polymorphisms with hip fracture and osteoporosis in postmenopausal women. Climacteric 22, 97-104, https://doi.org/10.1080/13697137.2018.1538339 (2019).

61. Daly, R., Saxon, L., Turner, C., Robling, A. \& Bass, S. J. B. The relationship between muscle size and bone geometry during growth and in response to exercise. Bone 34, 281-287 (2004).

62. Klein, C. S., Allman, B. L., Marsh, G. D. \& Rice, C. L. Muscle size, strength, and bone geometry in the upper limbs of young and old men. J. Gerontol. A Biol. Sci. Med. Sci 57, M455-459, https://doi.org/10.1093/gerona/57.7.m455 (2002)

63. Karasik, D. et al. Bivariate genome-wide linkage analysis of femoral bone traits and leg lean mass: Framingham study. J. Bone Min. Res. 24, 710-718, https://doi.org/10.1359/jbmr.081222 (2009).

64. Ronkainen, P. H. et al. Catechol-o-methyltransferase gene polymorphism is associated with skeletal muscle properties in older women alone and together with physical activity. PLoS One 3, e1819 (2008).

65. Salmen, T. et al. Relation of estrogen receptor-alpha gene polymorphism and hormone replacement therapy to fall risk and muscle strength in early postmenopausal women. Ann. Med. 34, 64-72 (2002).

66. Vandevyver, C. et al. Lack of association between estrogen receptor genotypes and bone mineral density, fracture history, or muscle strength in elderly women. J. Bone Min. Res. 14, 1576-1582 (1999).

67. Bjornstrom, L. \& Sjoberg, M. Mechanisms of estrogen receptor signaling: convergence of genomic and nongenomic actions on target genes. Mol. Endocrinol. 19, 833-842, https://doi.org/10.1210/me.2004-0486 (2005).

68. Yasar, P., Ayaz, G., User, S. D., Gupur, G. \& Muyan, M. Molecular mechanism of estrogen-estrogen receptor signaling. Reprod. Med. Biol. 16, 4-20, https://doi.org/10.1002/rmb2.12006 (2017).

69. Cauley, J. A. Estrogen and bone health in men and women. Steroids 99, 11-15, https://doi.org/10.1016/j.steroids.2014.12.010 (2015).

70. Gennari, L. et al. Estrogen receptor gene polymorphisms and the genetics of osteoporosis: a HuGE review. Am. J. Epidemiol. 161, 307-320, https://doi.org/10.1093/aje/kwi055 (2005).

71. Rolland, Y. M., Perry, H. M. 3rd, Patrick, P., Banks, W. A. \& Morley, J. E. Loss of appendicular muscle mass and loss of muscle strength in young postmenopausal women. J. Gerontol. A Biol. Sci. Med. Sci 62, 330-335, https://doi.org/10.1093/gerona/62.3.330 (2007).

72. Phillips, S. K., Sanderson, A. G., Birch, K., Bruce, S. A. \& Woledge, R. C. Changes in maximal voluntary force of human adductor pollicis muscle during the menstrual cycle. J. Physiol. 496(Pt 2), 551-557, https://doi.org/10.1113/jphysiol.1996.sp021706 (1996).

73. Liu, X.-G. et al. Genome-wide association and replication studies identified TRHR as an important gene for lean body mass. Am. J. Hum. Genet. 84, 418-423 (2009).

74. Miyamoto-Mikami, E. et al. Lack of association between genotype score and sprint/power performance in the Japanese population. J. Sci. Med. sport. 20, 98-103 (2017)

75. Fuku, N. et al. Exceptional longevity and muscle and fitness related genotypes: a functional in vitro analysis and case-control association replication study with SNPs THRH rs7832552, IL6 rs1800795, and ACSL1 rs6552828. Front. Aging Neurosci. 7, 59, https://doi.org/10.3389/fnagi.2015.00059 (2015).

76. Salvatore, D., Simonides, W. S., Dentice, M., Zavacki, A. M. \& Larsen, P. R. Thyroid hormones and skeletal muscle-new insights and potential implications. Nat. Rev. Endocrinol. 10, 206 (2014).

77. Janssen, I., Heymsfield, S. B., Baumgartner, R. N. \& Ross, R. Estimation of skeletal muscle mass by bioelectrical impedance analysis. J. Appl. Physiol. 89, 465-471, https://doi.org/10.1152/jappl.2000.89.2.465 (2000).

78. Faria, S. L., Faria, O. P., Cardeal, M. D. \& Ito, M. K. J. Os Validation study of multi-frequency bioelectrical impedance with dualenergy X-ray absorptiometry among obese patients. Obes. Surg. 24, 1476-1480 (2014).

79. Okasora, K. et al. Comparison of bioelectrical impedance analysis and dual energy X-ray absorptiometry for assessment of body composition in children. Pediatr. Int. 41, 121-125 (1999).

80. Lee, S. et al. Comparison between dual-energy X-ray absorptiometry and bioelectrical impedance analyses for accuracy in measuring whole body muscle mass and appendicular skeletal muscle mass. Nutrients 10, 738 (2018).

81. Wingo, B. C., Barry, V. G., Ellis, A. C. \& Gower, B. A. Comparison of segmental body composition estimated by bioelectrical impedance analysis and dual-energy X-ray absorptiometry. Clin. Nutr. ESPEN 28, 141-147, https://doi.org/10.1016/j. clnesp.2018.08.013 (2018).

82. Barrea, L. et al. Association between Mediterranean diet and hand grip strength in older adult women. 38, 721-729 (2019).

83. Hughes, D. C., Day, S. H., Ahmetov, I. I. \& Williams, A. G. Genetics of muscle strength and power: polygenic profile similarity limits skeletal muscle performance. J. Sports Sci. 29, 1425-1434, https://doi.org/10.1080/02640414.2011.597773 (2011).

84. Lee, W.-J. et al. Comparisons of sarcopenia defined by IWGS and EWGSOP criteria among older people: results from the I-Lan longitudinal aging study. J. Am. Med. Dir. Assoc. 14, 528. e521-528. e527 (2013).

85. Wang, H. et al. Estimation of prevalence of sarcopenia by using a new bioelectrical impedance analysis in Chinese communitydwelling elderly people. BMC geriatrics 16, 216 (2016).

86. Roberts, H. C. et al. A review of the measurement of grip strength in clinical and epidemiological studies: towards a standardised approach. Age Ageing 40, 423-429, https://doi.org/10.1093/ageing/afr051 (2011).

87. Villafane, J. H. et al. Reliability of the Handgrip Strength Test in Elderly Subjects With Parkinson Disease. Hand 11, 54-58, https:// doi.org/10.1177/1558944715614852 (2016).

88. Washburn, R., Ficker, J. J. J. O. S. M. \& Fitness, P. Physical Activity Scale for the Elderly (PASE): the relationship with activity measured by a portable accelerometer. 39, 336 (1999).

89. Tintle, N., Gordon, D., Van Bruggen, D. \& Finch, S. The cost effectiveness of duplicate genotyping for testing genetic association. Ann. Hum. Genet. 73, 370-378, https://doi.org/10.1111/j.1469-1809.2009.00516.x (2009).

90. Danilovic, D. L. et al. Height and bone mineral density in androgen insensitivity syndrome with mutations in the androgen receptor gene. Osteoporos. Int. 18, 369-374, https://doi.org/10.1007/s00198-006-0243-6 (2007).

91. Fischer, C. P. et al. Endurance training reduces the contraction-induced interleukin-6 mRNA expression in human skeletal muscle. Am. J. Physiol. Endocrinol. Metab. 287, E1189-1194, https://doi.org/10.1152/ajpendo.00206.2004 (2004).

92. Benjamini, Y. \& Hochberg, Y. Controlling the false discovery rate: a practical and powerful approach to multiple testing. Journal of the royal statistical society. Series B (Methodological), 289-300 (1995). 


\section{Acknowledgements}

This study was funded by the European Union as a part of MOVE-AGE, an Erasmus Mundus Joint Doctorate programme (2011-2015) for PK. The authors would like to thanks all the volunteers who participated in this study.

\section{Author contributions}

P.K. and C.I.M. designed research; P.K. and L.H. performed research; P.K., G.S., G.O.P., H.D., A.W., M.T. and C.I.M. helped P.K analysing and interpreting the results; P.K. wrote the manuscript; and L.H., G.S., G.O.P., H.D., A.W., M.T. and C.I.M. revised the manuscript.

\section{Competing interests}

The authors declare no competing interests.

\section{Additional information}

Supplementary information is available for this paper at https://doi.org/10.1038/s41598-020-59722-9.

Correspondence and requests for materials should be addressed to P.K.

Reprints and permissions information is available at www.nature.com/reprints.

Publisher's note Springer Nature remains neutral with regard to jurisdictional claims in published maps and institutional affiliations.

(c) (i) Open Access This article is licensed under a Creative Commons Attribution 4.0 International License, which permits use, sharing, adaptation, distribution and reproduction in any medium or format, as long as you give appropriate credit to the original author(s) and the source, provide a link to the Creative Commons license, and indicate if changes were made. The images or other third party material in this article are included in the article's Creative Commons license, unless indicated otherwise in a credit line to the material. If material is not included in the article's Creative Commons license and your intended use is not permitted by statutory regulation or exceeds the permitted use, you will need to obtain permission directly from the copyright holder. To view a copy of this license, visit http://creativecommons.org/licenses/by/4.0/.

(c) The Author(s) 2020 